\title{
Mechanics Modeling and Simulation of Flexible Cable on Spacecraft Considering Spatial Constraints
}

\author{
Bindi You, Jianmin Wen, Chuansong Zhai, Tianjiao Zheng and Binjiu Yang \\ School of Naval Architecture and Ocean Engineering, Harbin Institute of Technology, \\ 264209 Weihai, China \\ youbindi@hit.edu.cn,wenjm@hit.edu.cn
}

\begin{abstract}
According to the complicated spatial constraints of the spacecraft, combined with physical characteristics, a mechanics model of flexible cable is established considering the large deflection deformation. Then, influences of constraints on the cable spatial attitude, movement parameters and internal forces are found. The method of cable planning can be used in the virtual environment of the model considering the spatial constraints. Furthermore, the mechanics model is solved by numerical integration method. Finally, the variation laws of the cable parameters are analyzed, and the simulation results have verified the validity of the cable mechanical model.
\end{abstract}

Keywords: Flexible Cable, Spatial Constraints, Mechanics Model, Simulation

\section{Introduction}

Spacecrafts are unrepairable and are needed to complete the complex long-term work independently after launching [1]. Moreover, they have complex internal structures and spatial distributions and a large number of functional modules. So, electromagnetic compatibility, heat dissipation and seismic performance between cables and the system must be considered. The mechanical forces should be avoided when cables through the solid constraints. And, the degradations of physical performance should be avoided due to excessive winding and torsion [2]. Thus, the total mass of the assembled spacecrafts are rigorously restricted and operation reliability of the electrical system can be influenced by rational distributions of the cables [3].

Many methods of cable modeling focus on the motion descriptions. Some researchers have been interested in exploring the influences of the deformation energy on the cabling [3], others have concerned the influences of the physical properties on the cable models [4]. However, both large deflections of the cable and the complex spatial constraints of the spacecraft are not considered in these models. Moreover, they are difficult to meet the requirements of complex aerospace systems [5].

Generally, considering the influences of spatial geometry restraints and performance constraints on the cable planning and physical properties, mechanics model of the flexible cable is established. Then, the mechanics model is solved by numerical integration methods and variation laws of the cable parameters are obtained. All these are the theoretical foundations for subsequent path planning and feasibility analyses of the cables. 


\section{Mechanics Modeling with Spatial Constraints}

As shown in Figure 1, the fixed coordinate system $O-X Y Z$ is established in an arbitrary point $O$ of the assembly space of the cable, and local coordinate system $P$-xyz is established in an arbitrary point $P$ on the center line of the cable, where direction $z$ is the tangential direction of the cable. Then, according to an arbitrary section of the cable, mechanics analyses of the cable are performed. An arbitrary motion of the cable is performed with the forces and torques, where velocity is $v$, angular velocity is $\Omega$, and the angle of bending and torsion is $\omega$ in the arbitrary point $P$. Meanwhile, density $\rho$ and section area $S$ of the cable are defined. In the point $P$, principal vector and principal torque of the negative section with the internal force of the adjacent section are $-F$ and $-M$, and they are $(F+\Delta F)$ and $(M+\Delta M)$ in the adjacent point $P^{\prime}$. Moreover, environmental force and environmental torque of the unit length cable are $f$ and $m$, and the force and torque caused by weight of the unit length cable are $f_{g}$ and $m_{f g}$, respectively.

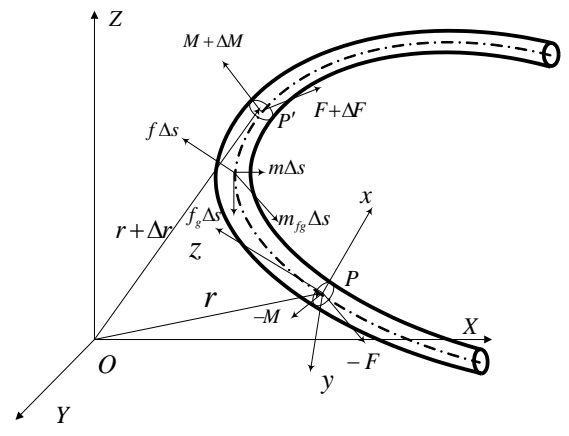

Figure 1. Mechanics modeling of the cable

Assuming that the cable is moving with certain velocity and angular velocity, dynamic equations of the cable are presented by using the Newton's second law and the Momentum Theorem.

$$
\begin{aligned}
& \Delta F+f \Delta s+f_{g} \Delta s=\rho S \Delta s \frac{\partial v}{\partial t} \\
& \Delta M+m \Delta s+m_{f g} \Delta s+\Delta r \times F=\Delta s \frac{\partial}{\partial t}(J \cdot \Omega)
\end{aligned}
$$

where $J$ is an inertia matrix.

$$
\begin{gathered}
J=\left(J_{x}, J_{y}, J_{z}\right) \\
J_{x}=J_{y}=\rho I_{x}=\rho I_{y}=\rho \frac{S^{2}}{4 \pi}, \quad J_{z}=\rho \frac{S^{2}}{2 \pi}
\end{gathered}
$$


Projections of equations in the directions of local coordinate system are expressed as

$$
\begin{aligned}
& \frac{\partial F_{x}}{\partial s}+\omega_{y} \times F_{z}-\omega_{z} \times F_{y}+f_{x}+\rho S\left(\mathrm{~g}_{\mathrm{x}}+\frac{\partial v_{x}}{\partial t}-\Omega_{y} \times v_{z}-\Omega_{z} \times v_{y}\right)=0 \\
& \frac{\partial F_{y}}{\partial s}+\omega_{z} \times F_{x}-\omega_{x} \times F_{z}+f_{y}+\rho S\left(\mathrm{~g}_{y}+\frac{\partial v_{y}}{\partial t}+\Omega_{x} \times v_{z}-\Omega_{z} \times v_{x}\right)=0 \\
& \frac{\partial F_{z}}{\partial s}+\omega_{x} \times F_{y}-\omega_{y} \times F_{x}+f_{z}+\rho S\left(\mathrm{~g}_{z}+\frac{\partial v_{z}}{\partial t}-\Omega_{x} \times v_{y}+\Omega_{y} \times v_{x}\right)=0 \\
& E I_{x} \frac{\partial \omega_{x}}{\partial s}+\omega_{y} \omega_{z}\left(G I_{z}-E I_{y}\right)+E I_{y} \omega_{y}^{0} \omega_{z}-G I_{z} \omega_{y} \omega_{z}^{0}+m_{x}+m_{f g x}-F_{y} \\
& -\frac{\partial}{\partial t}\left(J_{x} \cdot \Omega_{x}\right)-\Omega_{y} \times\left(J_{z} \cdot \Omega_{z}\right)+\Omega_{z} \times\left(J_{y} \cdot \Omega_{y}\right)=0 \\
& E I_{y} \frac{\partial \omega_{y}}{\partial s}+\omega_{x} \omega_{z}\left(E I_{x}-G I_{z}\right)+G I_{z} \omega_{x} \omega_{z}^{0}-E I_{x} \omega_{x}^{0} \omega_{z}+m_{y}+m_{f g y}+F_{x} \\
& -\frac{\partial}{\partial t}\left(J_{y} \cdot \Omega_{y}\right)+\Omega_{x} \times\left(J_{z} \cdot \Omega_{z}\right)-\Omega_{z} \times\left(J_{x} \cdot \Omega_{x}\right)=0 \\
& G I_{z} \frac{\partial \omega_{z}}{\partial s}+E \omega_{x} \omega_{y}\left(I_{y}-I_{x}\right)+E I_{x} \omega_{x}^{0} \omega_{y}-E I_{y} \omega_{x} \omega_{y}^{0}+m_{z}+m_{f g z} \\
& -\frac{\partial}{\partial t}\left(J_{z} \cdot \Omega_{z}\right)+\Omega_{y} \times\left(J_{x} \cdot \Omega_{x}\right)-\Omega_{x} \times\left(J_{y} \cdot \Omega_{y}\right)=0
\end{aligned}
$$

Here, the relationships between the angle of bending and torsion $\omega$ and the cable's angular velocity $\Omega$ should be explained clearly. Accordingly, $\omega$ is the infinitesimal angular displacement vector divided by the infinitesimal arc length increment $\Delta s$, that is, derivation to the arc length $s . \Omega$ is the infinitesimal angular displacement vector divided by the infinitesimal time increment $\Delta t$, that is, derivation to the time $t$. Thus, the relationship between $\omega$ and $\Omega$ is expressed as

$$
\frac{\tilde{\partial} \omega}{\partial t}=\frac{\tilde{\partial} \Omega}{\partial s}+\omega \times \Omega, \quad \frac{\tilde{\partial} v}{\partial s}+\omega \times v=\Omega \times z
$$

Projections of equation (6) in the directions of the local coordinate system are expressed as

$$
\begin{array}{ll}
\frac{\partial \omega_{x}}{\partial t}-\frac{\partial \Omega_{x}}{\partial s}+\omega_{z} \Omega_{y}-\omega_{y} \Omega_{z}=0, & \frac{\partial v_{x}}{\partial s}+\omega_{y} v_{z}-\omega_{z} v_{y}-\Omega_{y}=0 \\
\frac{\partial \omega_{y}}{\partial t}-\frac{\partial \Omega_{y}}{\partial s}+\omega_{x} \Omega_{z}-\omega_{z} \Omega_{x}=0, & \frac{\partial v_{y}}{\partial s}+\omega_{z} v_{x}-\omega_{x} v_{z}+\Omega_{x}=0 \\
\frac{\partial \omega_{z}}{\partial t}-\frac{\partial \Omega_{z}}{\partial s}+\omega_{y} \Omega_{x}-\omega_{x} \Omega_{y}=0, & \frac{\partial v_{z}}{\partial s}+\omega_{x} v_{y}-\omega_{y} v_{x}=0
\end{array}
$$

Equations (4), (5) and (7) are the dynamic model of the cable with constraints. Furthermore, in order to analyze the variation laws of the mechanics characters and the attitude of flexible cable with the constraints and the time, mechanics properties of some cables with special conditions are used to analyze the forces and motion laws of the cable clearly. 
By considering torsion motion of the cable, the cable motion can be simplified into the motion about the axis $z$. Namely, these equations can be rewritten as

$$
\begin{gathered}
\frac{\partial^{2} \omega_{z}}{\partial t^{2}}-\frac{\partial^{2} \Omega_{z}}{\partial s \partial t}+\frac{\partial\left(\omega_{y} \Omega_{x}-\omega_{x} \Omega_{y}\right)}{\partial t}=0 \\
G I_{z} \frac{\partial^{2} \omega_{z}}{\partial s^{2}}+\frac{\partial\left[E \omega_{x} \omega_{y}\left(I_{y}-I_{x}\right)\right]}{\partial s}+\frac{\partial\left(E I_{x} \omega_{x}^{0} \omega_{y}\right)}{\partial s}-\frac{\partial\left(E I_{y} \omega_{x} \omega_{y}^{0}\right)}{\partial s}+\frac{\partial m_{z}}{\partial s} \\
+\frac{\partial m_{f g z}}{\partial s}-J_{z} \frac{\partial^{2} \Omega_{z}}{\partial t \partial s}+\frac{\partial\left[\Omega_{y} \times\left(J_{x} \cdot \Omega_{x}\right)\right]}{\partial s}-\frac{\partial\left[\Omega_{x} \times\left(J_{y} \cdot \Omega_{y}\right)\right]}{\partial s}=0
\end{gathered}
$$

If coupling conditions of multiple planes are ignored, the cable bending is just in the plane$x o z$ by considering bending motion of the cable. Meanwhile, the distribution forces and initial angle of torsion of the cable are ignored, that is

$$
\varphi=0, \quad \tau=0, \quad \omega_{y}=\kappa, \quad \omega_{x}=\omega_{z}=\frac{\partial \varphi}{\partial s}=0
$$

The conditions obtained from equations (10) are substituted into the kinematics equations and dynamic equations, then

$$
\begin{gathered}
\frac{\partial \omega_{x}}{\partial t}=\frac{\partial \Omega_{y}}{\partial s}=\frac{\partial v_{z}}{\partial s}=0, \quad \omega_{x} \Omega_{y}=\omega_{x} v_{z}=0, \quad \frac{\partial v_{x}}{\partial s}-\Omega_{y}=0 \\
\frac{\partial F_{x}}{\partial s}+f_{x}+\rho S\left(g_{x}+\frac{\partial v_{x}}{\partial t}-\Omega_{y} \times v_{z}\right)=0 \\
\frac{\partial F_{y}}{\partial s}-\omega_{x} \times F_{z}+f_{y}+f_{g y}=0 \\
\frac{\partial F_{z}}{\partial s}+\omega_{x} \times F_{y}+f_{z}+\rho S\left(g_{z}+\frac{\partial v_{z}}{\partial t}+\Omega_{y} \times v_{x}\right)=0 \\
E I_{x} \frac{\partial \omega_{x}}{\partial s}-F_{y}=0
\end{gathered}
$$

In the single bending conditions, the local coordinate system of the cable is coincided with the position and attitude coordinate system, where direction $x$ is the normal direction, direction $y$ is the normal direction and direction $z$ is the tangent direction. Meanwhile, when the cable only keeps bending motion, the force in the normal direction is 0 and forces in other directions are single-valued functions of the angle of bending and torsion.

\section{Numerical Simulations of Mechanics Model with Spatial Motions}

Spatial motions are basic characteristic of the cabling, and the whole motion process of the cable can be considered as the combination of these spatial motions. These motion results are analyzed respectively.

Single bending motion: Conditions of the motion needed to satisfy are

$$
\begin{aligned}
& f_{y}=0 \\
& m_{x}+m_{g x}=m_{z}+m_{g z}=0
\end{aligned}
$$


In the special spatial constraints, the single bending motion can be achieved. Then, numerical integrations are performed, and variations of the angles of bending and torsion and the angular velocity of the cable can be obtained in the direction $z$.

As shown in Figure 2, the angles of bending and torsion and the angular velocities of the cable in the direction $x$ and direction $z$ are always 0 . And, they have components in the direction $y$. These agree with the spatial constraints of the cable introduced by equation (10).

The main title (on the first page) should begin $13 / 16$ inches (7 picas) from the top edge of the page, centered, and in Times New Roman 14-point, boldface type. Capitalize the first letter of nouns, pronouns, verbs, adjectives, and adverbs; do not capitalize articles, coordinate conjunctions, or prepositions (unless the title begins with such a word). Please initially capitalize only the first word in other titles, including section titles and first, second, and third-order headings (for example, "Titles and headings" — as in these guidelines). Leave two blank lines after the title.

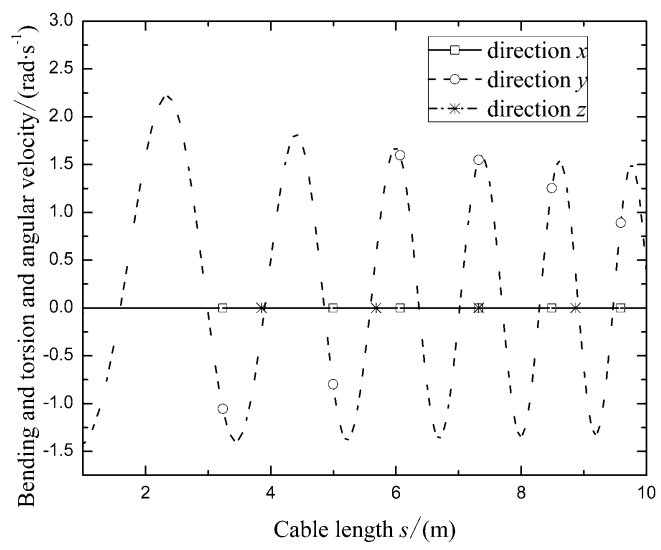

Figure 2. Angles of bending and torsion and angular velocity in single bending motion

As shown in Figure 3, when the cable is in the single bending state, motion velocity of the cable in the direction $y$ is always 0 . That is, the cable motion is always on a single plane, and the bending motion of the cable is the sum of velocities in the directions $x$ and $y$. Meanwhile, the cable velocity is increasing slowly with the stability of the cable force.

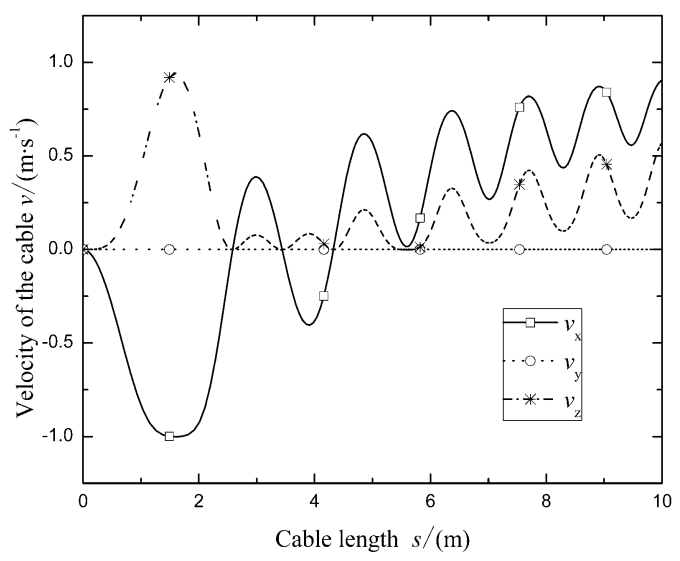

Figure 3. Velocity of the cable in single bending motion 


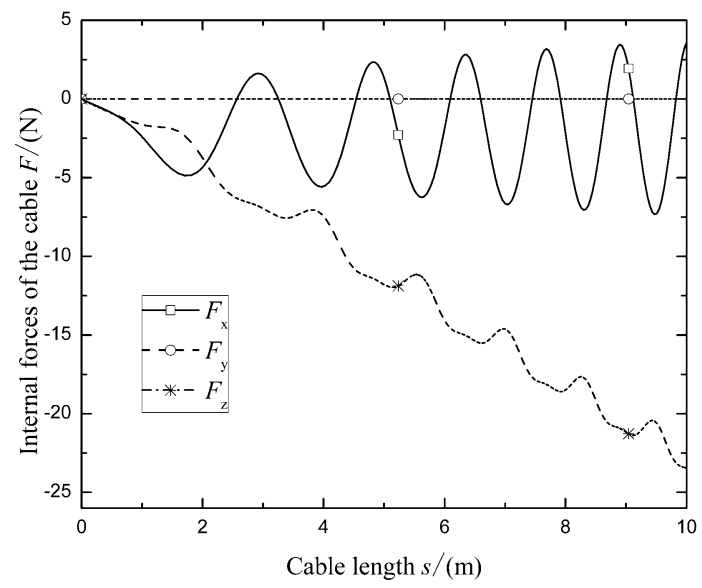

Figure 4. Internal forces of the cable in single bending motion

As shown in Figure 4, the deformation of the single bending motion of the cable relates to the forcing conditions of the cable. The force in the direction $y$ is always 0 and the cable motion is formed by forces in directions $x$ and $z$, and the cable can keep a certain acceleration.

Single torsion motion: Force conditions of the motion must be satisfied as

$$
\begin{aligned}
& f_{x}+f_{y}+f_{z}=0 \\
& m_{x}=m_{y}=0, \quad m_{g x}=m_{g y}=0
\end{aligned}
$$

When the cable is in the single torsion motion, the spatial attitude of the cable can be represented by its angles of bending and torsion, and motion state can be represented by the cable motion velocity. Then, numerical solutions of the kinematics equations and dynamic equations of the cable are obtained by using the numerical integration method. As shown in Figure 5, projections of the angles of bending and torsion of the cable in the directions $x$ and $y$ are 0 . And the angles of bending and torsion of the cable exist in the axis $z$, that is, the cable is obviously in the torsion motion.

Projections of the velocities and the internal forces of the cable in any direction are 0 .

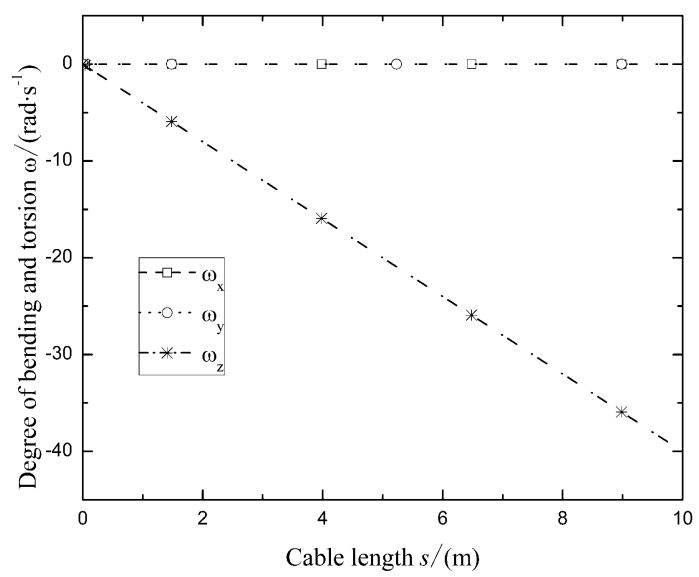

Figure 5. Angles of bending and torsion in single torsion motion 


\section{Conclusions}

In this paper, mechanics model of flexible cable considering spatial constraints is established. Then, parameters of the cable are analyzed by using numerical integration method, and variation laws of the cable motion are obtained.

(1) The kinematics and dynamic equations of the cable are developed considering the influences of the spatial constraint. Then, the mechanics model of the cable with spatial constraints is established.

(2) Considering the spatial motions of the cable individually, calculation results with the special conditions are presented. As shown in simulation results, the cable model proposed in this paper is valid. In virtual environment, when certain external forces are applied, the corresponding attitudes and motion conditions of the cable will play an important role in the future.

\section{Acknowledgements}

This paper is based on Project (Approval No. 51205079 and 51275108) Supported by National Science Foundation, and Project (HIT.KLOF.01507374) Supported by Key Laboratory Opening Funding of Technology of Micro-Spacecraft, and China Postdoctoral Science Foundation funded project (2013M541358), and Key Program of National Natural Science Foundation of Heilongiiang (No.ZD201309). The authors would like to thank the research grant from Spacecraft Dynamics Design and Simulation Lab (SDDSL) of HIT.

\section{References}

[1] S. Y. Sun and F. Yu, "Probe into Modular Tech of Electronic Equipments on Spacecrafts", Aerospace Standardization, vol. 3, (2010), pp. 18-24.

[2] V. A. Emil, "Cable Effects Study: Tangents, Rat Holes, and Valuable Results", 51st AIAA/ASME/ASCE/AHS/ASC Structure Dynamics, and Materi”, A Dissertation Submitted to China Academy of Engineering Physics for Master's Degree in Engineering, (2006).

[4] J. H. Liu, "Motional Cable Harness Physical Characteristic Oriented Modeling and Kinetic Simulation Technology in Virtual Environment”, Journal of Mechanical Engineering”, vol. 47, (2011), pp. 117-124.

[5] L. L. He, F. Y. Wei and F. J. Wang, "Research Status and Development Trend of Virtual Layout Technology", Machinery, vol. 46, (2008), pp. 67-70.

[6] Y. Xue, Y. Z. Liu and L. Q. Chen, “On Analytical Mechanics for a Super-thin Elastic Rod”, Chinese Journal of Theoretical and Applied Mechanics, vol. 37, (2005), pp. 485-493.

[7] Y. Xue, Y. Z. Liu and L. Q. Chen, "Methods of Analytical Mechanics for Dynamics of the Kirchhoff Elastic Rod”, Acta Physica Sinica, vol. 55, (2006), pp. 3845-3851. 
International Journal of Control and Automation Vol.7, No.3 (2014) 